\title{
Medical Assistance in Dying (MAiD) in Brain Cancer: Whose Autonomy Are We Respecting?
}

Keywords: Palliative care, Glioma, Dignity, Autonomy, Decision-making, Capacity, Advance care planning

doi: $10.1017 / \operatorname{cjn} .2021 .198$

Can J Neurol Sci. 2021; 48: 747-749

We lack effective and especially curative treatments for many brain cancers and unfortunately, for most adult patients, brain cancer is a terminal diagnosis. For those with lower grade tumours, the prognosis is less certain and survival may be measured in decades. Consequently, there is a need for early discussions in Neurooncology around supportive and palliative care that consider advance care planning and optimising function in the face of inevitable deterioration. For some patients, the choice of an early death is preferable to the fear of suffering and loss of independence that a slightly longer life might present. Medical assistance in dying (MAiD), also known as physician-assisted suicide (PAS) or voluntary-assisted dying (VAD), may be self-administered or clinician administered, depending on legislation. ${ }^{1}$ The practice remains controversial, and some have expressed concern that MAiD may be primarily driven by the inequity of access or failure to implement early and comprehensive palliative care as well as by potential social or financial vulnerability. ${ }^{2}$ Similarly, concerns exist regarding the inequity of access to MAiD itself, ${ }^{3}$ including in many faith-based health care settings where MAiD is unavailable. This polarising topic is overlaid by the potential negative impact of a brain cancer diagnosis on cognitive function and decision-making capacity that often goes unrecognised. ${ }^{4}$

Despite insufficient guidelines for competency assessment and decision-making for MAiD in Neuro-oncology, there is consensus that understanding both patient and clinician views is required. In this issue of the journal, Climans et al. reported on an international online survey of Neuro-oncologists identifying attitudes and perspectives on MAiD, including the assessment of decision-making capacity and the moral permissibility of the practice. ${ }^{5}$ Hypothetical scenarios were used to identify physician opinions about the eligibility of MAiD for certain patients. Accepting the limitations of low response rates (125/ $10,000)$, the study confirms differing opinions of Neuro-oncologists, although all acknowledge that capacity must be assessed carefully. Because most respondents were from jurisdictions in which MAiD is not yet legal, and only $16 \%$ had participated in the process, familiarity and provider experience may influence responses. This is supported by practitioners with increased time in clinical practice reporting fewer moral objections and increasing support from physicians in countries where MAiD has been implemented longer. ${ }^{6,7}$ Surveys of physicians in this context are also limited by non-random sampling as well as the framing of questions with 'yes-or-no' options. Additional understanding from this survey will be presented after qualitative interviews of survey respondents currently under evaluation.

MAiD has been lawful in some jurisdictions since the 1940s and in Canada since 2016. ${ }^{8}$ Since then, participation in MAiD has increased steadily each year and accounted for approximately $2.5 \%$ of all deaths in $2020,{ }^{9}$ and internationally this ranges from $0.3 \%$ to $4.6 \%{ }^{10}$ MAiD remains controversial and a topic of intense debate. Public interest is driven by concerns about suffering and the desire for patient autonomy, often motivated by highly visible and personal stories. In contrast, the physician perspective demonstrates less support, frequently highlighting concerns about misuse of MAiD in the context of financial and social vulnerability, as well as the dangers of the 'slippery slope', and multiple groups, including the American College of Physicians, have released position statements arguing against the support of MAiD. ${ }^{2,11}$ Despite these concerns, multiples cohorts have found MAiD recipients to be wealthier, well-educated, and more likely to be partnered, ${ }^{8,12-}$

15 suggesting that requests for MAiD are unlikely to be driven by social or financial inequity.

Brain cancer is unique, as a malignancy that affects the very essence or identity of an individual, due to the impact of the tumour on personality, cognitive function, and competence. Therefore, debate on MAiD in Neuro-oncology requires attention to selfidentity and maintaining the dignity of patients with brain cancer. Although pain and suffering are often considered drivers of requests for MAiD, more frequently the loss of dignity, autonomy and inability to enjoy life influences requests for MAiD. ${ }^{10,15}$ This concern raises questions about advance care planning and decision-making in the patient whose disease changes cognitive capacity such that they can no longer consent to MAiD. In this scenario, should we enact the wishes of the patient before their impairment or the 'new' patient before us who has been borne out of the disease? The issue of impaired decision-making capacity in patients with brain cancer may also be under-recognised, particularly in highgrade glioma (HGG). ${ }^{4}$ Assessment of this capacity is made doubly complex in brain tumour patients due to the prevalence of complicating symptoms including aphasia and intermittent impairment of consciousness. ${ }^{16}$ 
The popular focus on MAiD as the 'solution' to end-of-life suffering absorbs substantial political attention and funding, while neglecting most dying patients who may not consider it. In the context of brain cancer, a recent report from the State of Washington examining HGG patients' request for MAiD found only a minority of those who are prescribed elect to proceed with MAiD. ${ }^{17}$ Amongst palliative care providers, there is concern that MAiD will be further conflated in the public mind with palliative care (due to fear that the promotion of effective opioid analgesia in palliative care is 'euthanasia by stealth'), and this may dissuade patients who could reap substantial symptomatic benefits from accessing palliative care. ${ }^{18}$ Early, comprehensive palliative care support for brain cancer patients has been shown to result in high patient and carer satisfaction and improved quality of life, and to be highly cost-effective through reducing hospital readmission rates with uncontrolled symptoms or deterioration. ${ }^{19}$ It would be unfortunate if access to and funding of these goals were impacted by a disproportionate focus on MAiD.

Many arguments are advanced both in favour and against MAiD; however, the momentum internationally continues, and the intensity of debate shows no sign of abating. Detailed guidelines for decisionmaking in MAiD are indicated to support Neuro-oncologists in assessing competency while ensuring respect for patient autonomy and dignity. The question remains, can palliative care and MAiD co-exist and will improvements in and earlier introduction of palliative care in the brain cancer patient's journey be impeded by the further developments of MAiD? In the context of the terminal nature of most brain cancers, this study reminds us to engage early, frankly and often with our patients about their priorities and preferences for end-of-life care, to ensure that we can be advocates for and guardians of those wishes when their competence and autonomy are inevitably diminished by their disease.

\section{Disclosures}

The authors have no conflicts of interest to declare.

\section{Statement of Authorship}

All three authors equally contributed to design, manuscript preparation, and final approval.

James R. Whittle

Department of Medical Oncology, Peter MacCallum Cancer Centre, Melbourne, VIC, Australia

Sir Peter MacCallum Department of Oncology, The University of Melbourne, Melbourne, VIC, Australia

Cancer Biology and Stem Cells Division, The Walter and Eliza Hall Institute of Medical Research, Parkville, VIC, Australia

Department of Medical Biology, The University of Melbourne, Parkville, VIC, Australia

\section{Molly Williams \\ Children's Cancer Centre, The Royal Children's Hospital Melbourne, Parkville, VIC, Australia}

Victorian Paediatric Palliative Care Program, Parkville, VIC, Australia
David D. Eisenstat (1)

Children's Cancer Centre, The Royal Children's Hospital Melbourne, Parkville, VIC, Australia

Department of Paediatrics, The University of Melbourne, Parkville, VIC, Australia

Neuro-Oncology Laboratory, Murdoch Children's Research Institute, Parkville, VIC, Australia

Correspondence to: David D. Eisenstat, Children's Cancer Centre, The Royal Children's Hospital Melbourne, Parkville, VIC, Australia. Email: David.Eisenstat@rch.org.au

\section{REFERENCES}

1. Mroz S, Dierickx S, Deliens L, Cohen J, Chambaere K. Assisted dying around the world: a status quaestionis. Ann Palliat Med. 2021;10:3540-53.

2. Snyder Sulmasy L, Mueller PS, Ethics, Professionalism and Human Rights Committee of the American College of Physicians. Ethics and the legalization of physician-assisted suicide: an American College of Physicians position paper. Ann Intern Med. 2017;167:576-8.

3. Buchbinder M. Access to aid-in-dying in the United States: shifting the debate from rights to justice. Am J Public Health. 2018;108:754-9.

4. Kerrigan S, Erridge S, Liaquat I, Graham C, Grant R. Mental incapacity in patients undergoing neuro-oncologic treatment: a cross-sectional study. Neurology. 2014;83:537-41.

5. Climans SA, Mason WP, Edelstein K, Variath C, Bell JAH. Neurooncology clinicians' attitudes and perspectives on medical assistance in dying. Can J Neurol Sci. 2021;48:772-8. DOI 10.1017/ cjn.2021.186.

6. Kouwenhoven PS, Raijmakers NJ, van Delden JJ, et al. Opinions of health care professionals and the public after eight years of euthanasia legislation in the Netherlands: a mixed methods approach. Palliat Med. 2013;27:273-80.

7. Smets T, Cohen J, Bilsen J, Van Wesemael Y, Rurup ML, Deliens L. Attitudes and experiences of Belgian physicians regarding euthanasia practice and the euthanasia law. J Pain Symptom Manage. 2011;41:580-93.

8. Downar J, Fowler RA, Halko R, Huyer LD, Hill AD, Gibson JL. Early experience with medical assistance in dying in Ontario, Canada: a cohort study. CMAJ. 2020;192:E173-81.

9. Second annual report on medical assistance in dying in Canada. Health Canada; 2021. Available at: https://www.canada.ca/ content/dam/hc-sc/documents/services/medical-assistance-dying/ annual-report-2020/annual-report-2020-eng.pdf; accessed August 4, 2021

10. Emanuel EJ, Onwuteaka-Philipsen BD, Urwin JW, Cohen J. Attitudes and practices of euthanasia and physician-assisted suicide in the United States, Canada, and Europe. JAMA. 2016;316:79-90.

11. De Lima L, Woodruff R, Pettus K, et al. International Association for Hospice and Palliative Care Position statement: euthanasia and physician-assisted suicide. J Palliat Med. 2017;20:8-14

12. Steck N, Junker C, Maessen M, et al. Suicide assisted by right-to-die associations: a population based cohort study. Int J Epidemiol. 2014;43:614-22.

13. Chambaere K, Rietjens JA, Cohen J, et al. Is educational attainment related to end-of-life decision-making? A large post-mortem survey in Belgium. BMC Public Health. 2013;13:1055.

14. Dierickx S, Deliens L, Cohen J, Chambaere K. Comparison of the expression and granting of requests for euthanasia in Belgium in 2007 vs 2013. JAMA Intern Med. 2015;175: 1703-6. 
15. Oregon Death with Dignity Act. 2020 data summary. Oregon Public Health Division; 2021. Available at: https://www.oregon.gov/ oha/PH/PROVIDERPARTNERRESOURCES/EVALUATION RESEARCH/DEATHWITHDIGNITYACT/Documents/year23.pdf; accessed August 4, 2021.

16. Isenberg-Grzeda E, Sofie K, Larrivee EJ, Graber JJ. Legal assistance in dying for people with brain tumors. Ann Palliat Med. 2021;10:893-8.
17. Chamberlain M. Physician assisted suicide in high grade gliomas: a university-based practice perspective. Neuro Oncol. 2016;18: vi144.

18. Waran E, William L. Navigating the complexities of voluntary assisted dying in palliative care. Med J Aust. 2020;213:204-6 e1.

19. Pace A, Villani V, Di Pasquale A, et al. Home care for brain tumor patients. Neurooncol Pract. 2014;1:8-12. 